\title{
Foaming three-charge black holes
}

\author{
Iosif Bena, ${ }^{1}$ Chih-Wei Wang, ${ }^{2}$ and Nicholas P. Warner ${ }^{2,3}$ \\ ${ }^{1}$ School of Natural Sciences, Institute for Advanced Study, Einstein Dr., Princeton, New Jersey 08540, USA \\ ${ }^{2}$ Department of Physics and Astronomy, University of Southern California Los Angeles, California 90089-0484, USA \\ ${ }^{3}$ Department of Physics, Theory Division, CERN, Geneva, Switzerland
}

(Received 6 September 2006; published 29 June 2007)

\begin{abstract}
We find a very large set of smooth horizonless geometries that have the same charges and angular momenta as the five-dimensional, maximally spinning, three-charge, supersymmetric black hole $\left(J^{2}=\right.$ $\left.Q^{3}\right)$. Our solutions are constructed using a four-dimensional Gibbons-Hawking base space that has a very large number of two-cycles. The entropy of our solutions is proportional to $\sqrt{Q}$. In the same class of solutions we also find microstates corresponding to zero-entropy black rings, and these are related to the microstates of the black hole by continuous deformations.
\end{abstract}

DOI: $10.1103 /$ PhysRevD.75.124026

PACS numbers: 04.70.Dy, 04.65.+e, 11.25.-w

\section{INTRODUCTION}

It has been expected for some time that there are many supergravity solutions that have the same charges and angular momenta as a given three-charge black hole or black ring. However, the true extent of this degeneracy has only become apparent as the result of recent work [1,2]. In these papers it was shown that, through a geometric transition called a "bubbling transition," one can generate a huge number of smooth, horizonless supergravity solutions with a fixed set of asymptotic charges and angular momenta. All these solutions are dual to states in the D1-D5 conformal field theory (CFT), that describes black holes and black rings. The existence of such a large number of smooth horizonless solutions raises the distinct possibility that the typical CFT states that contribute to the black hole entropy are dual to such solutions. If this is true, it would imply that the black hole should be thought of as an "ensemble" of horizonless solutions; this would greatly deepen our understanding of black holes, and quantum gravity in general. A review of this can be found in $[3,4]$.

In this paper we focus on the "bubbling" of maximally rotating, three-charge supersymmetric (BPS) black holes and black rings in five dimensions. We find a very large number of smooth solutions that have exactly the same supersymmetries, size, charges, and angular momenta as these objects. Our solutions have no localized brane sources (all the charges come from fluxes wrapping nontrivial cycles), and no horizons, and thus can be thought of as (a subset of) the "microstates" of the corresponding classical objects.

The equations underlying our solutions were found in [5-8]. The "bubbling transition" is obtained by replacing the flat $\mathbb{R}^{4}$, which constitutes the four-dimensional spatial base of the five-dimensional black hole and black ring solutions, by a half-flat four-dimensional (hyper-Kähler) metric whose signature is allowed to change between +4 and -4 . For computational convenience, we take the base to be a four-dimensional Gibbons-Hawking $(\mathrm{GH})$ space with many centers and whose geometric charges are inte- gers of any sign. To be asymptotic to $\mathbb{R}^{4}$, the sum of the geometric charges must be equal to one, but near a negative charge the metric of the base will become negative-definite. As is shown in $[1,2,9]$, even if the fourdimensional base changes its overall sign, the warp factors ensure that the full 11-dimensional solution is smooth and Lorentzian. The GH base metrics have nontrivial twocycles that run between the geometric charges; these are the "bubbles," and the bubbling transition may be thought of as a process of pair creation, separation, and decomposition of geometric charge. In this way the charges of the branes are "topologically partitioned" amongst the bubbles.

The three-charge, rotating, BPS black hole in five dimensions (also known as the BMPV black hole) [10] has two equal angular momenta that are bounded above by the square root of the product of the charges: $J_{1}=J_{2} \leq$ $\sqrt{Q_{1} \bar{Q}_{5} Q_{P}}$. The maximally spinning (or zero-entropy) BPS black hole saturates this bound. We show that if we have a large enough number of bubbles (or cycles), with fluxes of comparable magnitude, one always obtains $J_{1}^{2}=$ $J_{2}^{2} \approx Q_{1} Q_{5} Q_{P}$ in the limit when the number of bubbles becomes large. In fact, if the number of centers is large enough, it appears much more difficult to find a solution that does not have $J_{1}^{2}=J_{2}^{2} \approx Q_{1} Q_{5} Q_{P}$. To violate this, some of the fluxes have to be very much larger than others.

If the fluxes on all the cycles that end on one of the positive-charge GH points are very much larger than all the other fluxes, then that GH point will move very far from the rest of the GH points, and the solutions become one of the microstates of zero-entropy BPS black rings $[7,8,11-13] .{ }^{1}$ In fact, there is no sharp distinction between black ring microstates and black hole microstates: When the positive-

\footnotetext{
${ }^{1}$ The solutions that we consider here are more general than the zero-entropy black ring microstates constructed in [1]: The latter solutions involved a single geometric transition that resulted in two bubbles, while here we consider clusters of large numbers of bubbles. Other black ring microstate geometries have been constructed in [14].
} 
charge GH point is very far away from the others then the solution is clearly a ring microstate and when this point is in a cluster with all the others then the solution is clearly a black hole microstate. At intermediate separations such classical distinctions are not meaningful.

\section{A REVIEW OF THE BUBBLING SOLUTION}

The 11-dimensional solutions that have the same charges and supersymmetries as a three-charge BPS black hole have the metric [5-8]:

$$
\begin{aligned}
d s_{11}^{2}= & -\left(\frac{1}{Z_{1} Z_{2} Z_{3}}\right)^{2 / 3}(d t+k)^{2}+\left(Z_{1} Z_{2} Z_{3}\right)^{1 / 3} h_{m n} d x^{m} d x^{n} \\
& +\left(\frac{Z_{2} Z_{3}}{Z_{1}^{2}}\right)^{1 / 3}\left(d x_{1}^{2}+d x_{2}^{2}\right)+\left(\frac{Z_{1} Z_{3}}{Z_{2}^{2}}\right)^{1 / 3}\left(d x_{3}^{2}+d x_{4}^{2}\right) \\
& +\left(\frac{Z_{1} Z_{2}}{Z_{3}^{2}}\right)^{1 / 3}\left(d x_{5}^{2}+d x_{6}^{2}\right),
\end{aligned}
$$

where the four-dimensional base metric $h_{m n} d x^{m} d x^{n}$ is hyper-Kähler. In $[1,2,9]$ it was shown that when $h_{m n} d x^{m} d x^{n}$ is a Gibbons-Hawking (GH) metric:

$$
h_{m n} d x^{m} d x^{n}=V^{-1}(d \psi+\vec{A} \cdot d \vec{y})^{2}+V(d \vec{y} \cdot d \vec{y}),
$$

with $\vec{y} \in \mathbb{R}^{3}$ and $\vec{\nabla} \times \vec{A}=\vec{\nabla} V$, one can obtain a smooth 11-dimensional metric even if the geometric charges in $V$ are negative. Therefore, we take:

$$
V=\sum_{j=1}^{N} \frac{q_{j}}{r_{j}},
$$

with $r_{j} \equiv\left|\vec{y}-\vec{y}^{(j)}\right|$. To be regular at $r_{j}=0$ one must choose $q_{j} \in \mathbb{Z}$, and to be asymptotic to flat $\mathbb{R}^{4}$ one must take $q_{0} \equiv \sum_{j=1}^{N} q_{j}=1$. The GH metric has nontrivial twocycles (the bubbles), $\Delta_{i j}$, defined by the fiber coordinate $\psi$ and any line running between a pair of GH points, $\vec{y}^{(i)}$ and $\vec{y}^{(j)}$.

The background three-form potentials in the 11dimensional solution are defined via vector potentials in the four-dimensional GH base,

$$
\begin{aligned}
\mathcal{A}= & A^{(1)} \wedge d x_{1} \wedge d x_{2}+A^{(2)} \wedge d x_{3} \wedge d x_{4} \\
& +A^{(3)} \wedge d x_{5} \wedge d x_{6},
\end{aligned}
$$

Supersymmetry requires the "dipole field strengths:"

$$
\Theta^{(I)} \equiv d A^{(I)}+d\left(\frac{d t+k}{Z_{I}}\right)
$$

to be self-dual. One can easily show that if the base is Gibbons-Hawking, the $\Theta^{I}$ are given by

$$
\begin{aligned}
\Theta^{(I)}= & -\sum_{a=1}^{3}\left[\partial_{a}\left(V^{-1} K^{I}\right)\right]\left[(d \psi+A) \wedge d y^{a}\right. \\
& \left.+\frac{1}{2} V \epsilon_{a b c} d y^{b} \wedge d y^{c}\right],
\end{aligned}
$$

where $K^{I}$ is harmonic in the $\mathbb{R}^{3}$ base of the GH space. To have completely nonsingular solutions one must choose these to be sourced only at the singular points of $V$ :

$$
K^{I}=\sum_{j=1}^{N} \frac{k_{j}^{I}}{r_{j}}
$$

One then finds that the two-form fluxes through $\Delta_{i j}$ are given by:

$$
\Pi_{i j}^{(I)}=\left(\frac{k_{j}^{I}}{q_{j}}-\frac{k_{i}^{I}}{q_{i}}\right)
$$

Having chosen the flux parameters, $k_{j}^{I}$, the form of smooth horizonless solutions is completely fixed by the asymptotics and by requiring the absence of singular sources. See [1,2] for details. One further important physical ingredient in the solution is to avoid Dirac-Misner strings and closed timelike curves (CTC's). This requires one to impose the bubble equations ${ }^{2}[1,2]$ :

$$
\frac{1}{6} C_{I J K} \sum_{\substack{j=1 \\ j \neq i}}^{N} \Pi_{i j}^{(I)} \Pi_{i j}^{(J)} \Pi_{i j}^{(K)} \frac{q_{i} q_{j}}{r_{i j}}=-\sum_{I=1}^{3}\left(k_{i}^{I}-q_{i} \sum_{j=1}^{N} k_{j}^{I}\right),
$$

for $i=1, \ldots, N$, and where $r_{i j} \equiv\left|\vec{y}^{(i)}-\vec{y}^{(j)}\right|$. The bubble equations are required to remove CTC's in specific, "potentially dangerous" limits, and seem to solve the problem globally in a number of solutions. However, in general, the absence of CTC's requires that one ensure that the following are globally true:

$$
V Z_{I} \geq 0, \quad Z_{1} Z_{2} Z_{3} V-\mu^{2} V^{2} \geq 0,
$$

where $\mu$ is defined in [1].

The charges of the solution are $[2,19]^{3}$ :

$$
Q_{I}=-2 C_{I J K} \sum_{j=1}^{N} q_{j}^{-1} \tilde{k}_{j}^{J} \tilde{k}_{j}^{K},
$$

where

$$
\tilde{k}_{j}^{I} \equiv k_{j}^{I}-q_{j} N k_{0}^{I} \quad \text { and } \quad k_{0}^{I} \equiv \frac{1}{N} \sum_{j=1}^{N} k_{j}^{I} .
$$

The angular momenta are given by:

\footnotetext{
${ }^{2}$ One can add a constant to $V$ in (2.3), and reduce the resulting smooth five-dimensional solution to a singular four-dimensional multi-black hole solution of the type explored in $[15,16]$. The "bubble equations" are then equivalent to the "integrability conditions" of $[15,16]$. Other asymptotically four-dimensional configurations that are smooth in five dimensions have been explored in $[17,18]$.

${ }^{3}$ These results appear to differ by some factors of two compared to those of [2]. This is because our conventions are those of [1], which use a different normalization of the two-form fields.
} 


$$
\begin{aligned}
& J_{R} \equiv J_{1}+J_{2}=\frac{4}{3} C_{I J K} \sum_{j=1}^{N} q_{j}^{-2} \tilde{k}_{j}^{I} \tilde{k}_{j}^{J} \tilde{k}_{j}^{K}, \\
& J_{L} \equiv J_{1}-J_{2}=8|\vec{D}|,
\end{aligned}
$$

where

$$
\vec{D}_{j} \equiv \sum_{I} \tilde{k}_{j}^{I} \vec{y}^{(j)}, \quad \vec{D} \equiv \sum_{j=1}^{N} \vec{D}_{j}
$$

The bubble equations do not seem to allow straightforward and intuitive solutions. It is therefore rather natural to see what we can deduce either without them, or having made some extremely simple approximations.

\section{THE FOAMING BPS BLACK HOLE}

We first consider a configuration of $N$ GH centers that lie in a single "blob" and that all have roughly the same flux parameters to leading order in $N$. To keep the computation simple we take $N=2 M+1$ to be odd, and consider a distribution with $q_{j}=(-1)^{j+1}$. We can take all of $k_{i}^{I}$ to be positive numbers, and we will assume that their variations about their mean value are of the same order as the mean value:

$$
k_{j}^{I}=k_{0}^{I}(1+\mathcal{O}(1))
$$

where $k_{0}^{I}$ is defined in (2.12). Using (2.11) we find the charges to be:

$$
\begin{aligned}
Q_{I} & =2 C_{I J K}\left(N^{2} k_{0}^{J} k_{0}^{K}-\sum_{j} k_{j}^{J} k_{j}^{K} q_{j}^{-1}\right) \\
& \approx 2 C_{I J K}\left(N^{2}+\mathcal{O}(1)\right) k_{0}^{J} k_{0}^{K},
\end{aligned}
$$

where we used (3.1) and the fact that $\left|q_{i}\right|=1$ only in the last step. If $\left|q_{j}\right| \neq 1$ then the last term would yield a correction of, at most, $\mathcal{O}(N)$.

We can make a similar computation for the angular momenta using (2.13):

$$
\begin{aligned}
J_{R}= & \frac{4}{3} C_{I J K}\left(2 N^{3} k_{0}^{I} k_{0}^{J} k_{0}^{K}+\sum_{j} q_{j}^{-2} k_{j}^{I} k_{j}^{J} k_{j}^{K}\right. \\
& \left.-3 N k_{0}^{I} \sum_{j} q_{j}^{-1} k_{j}^{J} k_{j}^{K}\right) \\
\approx & \frac{4}{3} C_{I J K}\left(2 N^{3}+\mathcal{O}(N)\right) k_{0}^{I} k_{0}^{J} k_{0}^{K},
\end{aligned}
$$

where we have again used the fact that, for a "wellbehaved" distribution of positive $k_{i}^{I}$ with $\left|q_{j}\right|=1$ and $q_{0}=1$ one has:

$$
\begin{aligned}
\sum_{i} q_{i}^{-1} k_{i}^{J} k_{i}^{K} & =\sum_{i} q_{i} k_{i}^{J} k_{i}^{K} \approx k_{0}^{J} k_{0}^{K}, \\
\sum_{i} k_{i}^{I} k_{i}^{J} k_{i}^{K} & \approx N k_{0}^{I} k_{0}^{J} k_{0}^{K} .
\end{aligned}
$$

Once again, if $\left|q_{j}\right| \neq 1$ then (3.3) would remain true to $\mathcal{O}\left(N^{2}\right)$.

These results did not involve the solution of the "bubble equations." However, the formula for $J_{L}$ does depend rather nontrivially on the details of such a solution. One can also estimate the magnitude of $J_{L}$ in a few increasingly more complicated situations, and thereby argue that it is typically subleading in the large- $N$ limit.

Clearly, any configuration with three independent $\mathbb{Z}_{2}$ reflection symmetries will have $\vec{D}=0$. Hence, if one distributes all the points on a line (this would give $U(1) \times$ $U(1)$ invariant microstates), and if all the $k_{i}^{I}$ are equal, then $\vec{D}=0$. If one now takes the points on the line, but with different $k_{i}^{I}$, one can still exactly calculate $\vec{D}$ [19], and find it to be subleading. Intuitively this is happening because the largest part of the contributions to $\vec{D}$ from the positive charge centers is cancelled by the contribution from the nearby negative charge centers. The same intuition can be used to argue that the only way to get a large $|\vec{D}|$ from a compact blob is to "bias it" in terms of the locations of, and fluxes on, the positive and negative GH centers. This is basically what happens in the solutions of [9].

If we now focus on $\mathrm{M}$ theory on $T^{6}$, in the large- $N$ limit, we have

$$
\begin{aligned}
& Q_{1} \approx 4 N^{2} k_{0}^{2} k_{0}^{3}, \quad Q_{2} \approx 4 N^{2} k_{0}^{1} k_{0}^{3}, \\
& Q_{3} \approx 4 N^{2} k_{0}^{1} k_{0}^{2} ; \quad J_{R} \approx 16 N^{3} k_{0}^{1} k_{0}^{2} k_{0}^{3},
\end{aligned}
$$

and hence $J_{R}^{2} \approx 4 Q_{1} Q_{2} Q_{3}$, exactly as one finds for the maximally spinning BMPV black hole. One can also estimate how much the charges of our configurations differ from those of the maximally spinning BMPV black hole, and one finds that

$$
\frac{J_{R}^{2}}{4 Q_{1} Q_{2} Q_{3}}-1 \sim O\left(\frac{1}{N^{2}}\right) .
$$

Interestingly enough, the value of $J_{R}$ is slightly bigger than $\sqrt{4 Q_{1} Q_{2} Q_{3}}$. However, this is not a problem because in the classical limit, when the black hole solution is valid, these become equal. One can also argue that a classical black hole with zero horizon area will receive higher-order curvature corrections, which typically cause a positive increase in the horizon area; hence the $S=0$ microstates can have $J_{R}$ slightly larger than the maximal classically allowed value in order to compensate for this increase.

One can also easily see that our formulae reproduce the charges and angular momentum of the maximally spinning BPS black hole in any five-dimensional $U(1)^{k}$ supergravity. If we define $Y^{I}$ using 


$$
Q_{I} \equiv \frac{1}{2} C_{I J K} Y^{J} Y^{K},
$$

then the maximal angular momentum of this black hole is [20]:

$$
J_{R}=\frac{1}{3} C_{I J K} Y^{I} Y^{J} Y^{K}
$$

which clearly agrees with (3.2) and (3.3) in the large- $N$ limit.

\section{THE FOAMING BLACK RING}

The next-simplest configuration is a blob of total GH charge zero with a single, very distant point of GH charge +1 . One can view this as a continuous deformation of the configuration considered above. Again we will assume $N$ to be odd, and take the GH charge distribution to be $q_{j}=$ $(-1)^{j+1}$, with the distant charge being the $N$ th $\mathrm{GH}$ charge.

To have the $N$ th GH charge far from the blob means that all the two-cycles, $\Delta_{j N}$ must support a very large flux compared to the fluxes on the $\Delta_{i j}$ for $i, j<N$. Indeed, the former fluxes must be of order $N$ larger than the latter. To implement this, we take:

$$
\begin{gathered}
k_{j}^{I}=a_{0}^{I}(1+\mathcal{O}(1)), \quad j=1, \ldots, N-1, \\
k_{N}^{I}=-b_{0}^{I} N,
\end{gathered}
$$

where the $b_{0}^{I}$ are parameters of the same order as $a_{0}^{I}$, and the $a_{0}^{I}$ are defined as averages of the flux parameters over the blob:

$$
a_{0}^{I} \equiv \frac{1}{N-1} \sum_{j=1}^{N-1} k_{j}^{I} .
$$

The fluxes of this configuration are then:

$$
\begin{gathered}
\Pi_{i j}^{(I)}=\left(\frac{k_{j}^{I}}{q_{j}}-\frac{k_{i}^{I}}{q_{i}}\right), \quad \Pi_{i N}^{(I)}=-\Pi_{N i}^{(I)}=-\left(\frac{k_{i}^{I}}{q_{i}}+N b_{0}^{I}\right), \\
i, j=1, \ldots, N-1 .
\end{gathered}
$$

For this configuration one has:

$$
\begin{gathered}
k_{0}^{I}=\frac{(N-1)}{N} a_{0}^{I}-b_{0}^{I}, \quad \tilde{k}_{N}^{I}=-(N-1) a_{0}^{I}, \\
\tilde{k}_{j}^{I}=a_{j}^{I}+q_{j}\left(N b_{0}^{I}-(N-1) a_{0}^{I}\right), \quad j=1, \ldots, N-1 .
\end{gathered}
$$

Motivated by the bubbling black ring of [1], define the physical parameters:

$$
d^{I} \equiv 2(N-1) a_{0}^{I}, \quad f^{I} \equiv(N-1) a_{0}^{I}-2 N b_{0}^{I} .
$$

Then exactly the same arguments as those employed earlier lead to the following expressions for the charges and angular momentum:

$$
\begin{aligned}
Q_{I}= & \left(1+\mathcal{O}\left(N^{-2}\right)\right) C_{I J K} d^{J} f^{K}, \\
J_{R}= & \left(1+\mathcal{O}\left(N^{-2}\right)\right)\left(\frac{1}{2} C_{I J K}\left(d^{I} d^{J} f^{K}+f^{I} f^{J} d^{K}\right)\right. \\
& \left.-\frac{1}{24} C_{I J K} d^{I} d^{J} d^{K}\right) .
\end{aligned}
$$

Since the $N$ th point is far from the blob, we can take $r_{i N} \approx r_{0}$ and then the $N$ th bubble equation reduces to:

$$
\begin{aligned}
& \frac{1}{6} C_{I J K} \sum_{j=1}^{N-1}\left(\frac{a_{j}^{I}}{q_{j}}+N b_{0}^{I}\right)\left(\frac{a_{j}^{J}}{q_{j}}+N b_{0}^{J}\right)\left(\frac{a_{j}^{K}}{q_{j}}+N b_{0}^{K}\right) \frac{q_{j}}{r_{0}} \\
& =(N-1) \sum_{I} a^{I} .
\end{aligned}
$$

To leading order in $N$ this means that the distance from the blob to the $N$ th point, $r_{0}$, in the GH space is given by:

$$
\begin{aligned}
r_{0} & \approx \frac{1}{2} N^{2}\left[\sum_{I} a^{I}\right]^{-1} C_{I J K} a_{0}^{I} b_{0}^{J} b_{0}^{K} \\
& =\frac{1}{32}\left[\sum_{I} d^{I}\right]^{-1} C_{I J K} d^{I}\left(2 f^{J}-d^{J}\right)\left(2 f^{K}-d^{K}\right) .
\end{aligned}
$$

Finally, considering the dipoles, (2.14), it is evident that to leading order in $N, \vec{D}$ is dominated by the contribution coming from the $N$ th point and that:

$$
\begin{aligned}
J_{1}-J_{2} & =8|\vec{D}| \approx 8(N-1)\left(\sum_{I} a_{0}^{I}\right) r_{0} \\
& \approx 4 N^{2}(N-1) C_{I J K} a_{0}^{I} b_{0}^{J} b_{0}^{K} \\
& =\frac{1}{8} C_{I J K} d^{I}\left(2 f^{J}-d^{J}\right)\left(2 f^{K}-d^{K}\right) .
\end{aligned}
$$

One can easily verify that these results perfectly match the properties of the bubbled supertube found in [1]. Thus the blob considered here has exactly the same size, angular momenta, and charges as the zero-entropy three-charge black ring.

\section{THE ENTROPY OF THE FOAM}

As we have seen, when the number of centers is very large, getting a solution with $J_{1}^{2}=J_{2}^{2}=Q_{1} Q_{2} Q_{3}$ is not hard. In fact, it seems to be much harder to get anything else, unless one drastically increases the flux parameters $k_{i}^{I}$ on some subset of the cycles. It is natural therefore to ask how many bubbled black hole configurations are there for some given total charges.

If we work in M theory on $T^{6}$, then (3.5) implies that

$$
\sum k_{i}^{1}=\frac{1}{2} \sqrt{\frac{\overline{Q_{2} Q_{3}}}{Q_{1}}},
$$

and similarly for $\sum k_{i}^{2}$ and $\sum k_{i}^{3}$. Note that these relations depend upon neither the number nor the charges of the GH points. Hence, there is a nontrivial entropy coming simply from the many possibilities of choosing the positive $k_{i}^{I}$ subject to constraints of the form (5.1). Since this entropy 
comes entirely from the combinatorics of laying out quantized fluxes on topologically nontrivial cycles, we will refer to it as the topological entropy.

\section{A. Topological entropy}

The parameters $k_{i}^{I}$ are half-integers (up to a gauge shift induced by $K^{I} \rightarrow K^{I}+c^{I} V$ ). This can be seen most easily from their relation to the integer black ring dipole charge (4.5), and can be also derived by requiring the integral of $\Theta^{(I)}$ over the $S^{2}$ surrounding any GH center in $\mathbb{R}^{3}$ to be quantized. The entropy in choosing the positive halfintegers $k_{i}^{1}$ is therefore:

$$
S=2 \pi \sqrt{\frac{1}{6}\left(\frac{Q_{2} Q_{3}}{Q_{1}}\right)^{1 / 2}} .
$$

Naively there should be similar factors coming from partitioning $k_{i}^{2}$ and $k_{i}^{3}$, which leads to:

$$
\begin{aligned}
S_{\text {topological }}= & 2 \pi\left(\sqrt{\frac{1}{6}\left(\frac{Q_{2} Q_{3}}{Q_{1}}\right)^{1 / 2}}+\sqrt{\frac{1}{6}\left(\frac{Q_{1} Q_{2}}{Q_{3}}\right)^{1 / 2}}\right. \\
& \left.+\sqrt{\frac{1}{6}\left(\frac{Q_{1} Q_{3}}{Q_{2}}\right)^{1 / 2}}\right) .
\end{aligned}
$$

There are, however, some subtleties. First, the partitioning of $k_{i}^{1}, k_{i}^{2}$, and $k_{i}^{3}$ is not completely independent. A bubble will collapse unless all three fluxes are nonzero, and so we should count the ways of having nonzero partitions of all the $k_{i}^{I}$ over $N$ bubbles and then sum over $N$. One can show that this constraint contributes only subleading $(\log (Q))$ corrections to the entropy (see [19] for details). Second, given the $k_{j}^{1}$, there are also further constraints on $k_{j}^{2}$ and $k_{j}^{3}$ imposed by the global absence of CTC's. These conditions are somewhat more difficult to handle but we believe that the bubble equations, combined with some suitable positivity conditions on the fluxes, will suffice to guarantee the conditions in (2.10), and hence that (5.3) is correct to leading order. Independent of these subtleties and the constraints on $k_{j}^{2}$ and $k_{j}^{3}$ for a given set of $k_{j}^{1}$, we see from (5.2) alone that the topological entropy grows as $Q^{1 / 4}$.

If the topological entropy dominates then the "typical" microstate has of order $Q^{1 / 4}$ centers, with flux parameters of order $Q^{1 / 4}$ on each. One can estimate the typical distance (measured in the full, physical 11-dimensional metric) between two adjacent points and one finds that it is of order $Q^{1 / 4}$, which is parametrically larger than the Planck scale.

There exists another possible source of topological entropy. We have shown that, for a large number of bubbles, the charges (3.2) and angular momenta (3.3) only depend on the $k_{i}^{I}$, and not on the $q_{i}$, and so it appears that any "well-behaved" distribution of $q_{i}$ will give a good microstate. Since the $q_{i}$ can be arbitrary positive and negative integers, the number of such configurations is infinite. However, one must recall that the quantization of fluxes on the two-cycles running between any two GH centers implies that the $\Pi_{i j}^{(I)}$ are half-integers. ${ }^{4}$ Therefore, for a certain distribution of the $k_{i}^{I}$, the $q_{i}$ can only take a finite set of values and so the contribution to the topological entropy coming from having $\left|q_{i}\right| \neq 1$ is subleading. Alternatively, this quantization rule means that, up to a gauge shift, the $k_{j}^{I} / q_{j}$ are half-integers, and so there are no "frozen" $\mathrm{GH}$ points: It is possible to separate $\mathrm{GH}$ charges at a point with $\left|q_{j}\right| \neq 1$ and partition the fluxes while respecting the quantization rules. Thus the configurations with $\left|q_{j}\right| \neq 1$ are merely special points in the phase space of solutions with $\left|q_{j}\right|=1$.

\section{B. Moduli space entropy}

Another potentially large source of entropy arises from the moduli associated with the possible locations of the $\mathrm{GH}$ centers: There are $3(N-1)$ such positions (excluding the center of mass) and $(N-1)$ constraints from the bubble equations, (2.9). In [1] this moduli space was analyzed in simple examples and it was found that, in the first approximation, each cycle running between adjacent positive and negative centers behaves like an almost rigid rod. The most generic configuration is one in which all the rods have different orientations. It is not clear how to quantize this moduli space and thereby compute the entropy, but one can give a very rough estimate.

The length $L$ in $\mathbb{R}^{3}$ of a rod, or bubble, (measured in the full, physical 11-dimensional metric) is of order the flux $k$ through the bubble. The phase space of such a rod should be proportional to the number of Planck-size regions on a two-sphere of radius $L$, which is approximately $L^{2} / l_{p}^{2} \sim$ $k^{2}$. There are $N \sim \sqrt{Q} / k$ rods in such a configuration, and so the number of states of charge $Q$ and flux $k$ is approximately:

$$
d(Q, k) \sim k^{2(\sqrt{Q} / k)} .
$$

As a function of $k$, this has a maximum at $k=e$ (in Planck units) and this gives a contribution to the entropy of

$$
S_{\text {moduli }} \sim \frac{2}{e} \sqrt{Q}
$$

Our rough estimate indicates therefore that this contribution to the entropy is dominated by Planck scale bubbles, and is much larger than the topological entropy. It remains to be seen whether this rather naive analysis of the quantum moduli space is valid and whether the description of the bubbles in terms of "rods" is sufficiently accurate to

\footnotetext{
${ }^{4}$ This quantization condition is somewhat more stringent than that suggested in [2]. One can also try to estimate the topological entropy using the quantization in [2]. At first glace it appears to only differ from (5.2) by terms that are subleading in the charges.
} 
determine the dominant contribution to the entropy. A more involved analysis of the moduli space entropy for four-dimensional multi-black hole solutions has been performed in [21]. It would be interesting to see if one could adapt and extend that analysis to the present problem.

While both the moduli space entropy and the topological entropy are large, they are not large enough to be classically visible in supergravity; this is not surprising, since the classical entropy of the black hole with the same charges is zero. $^{5}$

Finally, we note that the geometry of the distribution of points in the $\mathbb{R}^{3}$ of the GH base can be deceptive when it comes to understanding the full geometry of the microstates. Even if all the points are very close together (in the metric in $\mathbb{R}^{3}$ ) and appear to be sitting on a long line or in a blob, the warp factors serve to blow up the bubbles and expand the space-time metric to create the familiar BPS black hole throat. Indeed, if one draws an $S^{2}$ around all the GH points (and thus an $S^{3}$ around the bubbles), then outside this sphere the geometry will look very much like the throat of a rotating BPS black hole. This is because the formation of the throat is a consequence of the net charges within the circumscribing sphere and these are the same for the bubbled microstates and for the black hole. The only difference is inside this sphere: The black hole has an infinite throat, while the microstate geometries have a finite one.

In a forthcoming paper [19] we will examine the appearance of the bubbled geometries in more detail and perform several gedanken experiments involving merging black ring foams and black hole foams and comparing with the results of [22]. This will further establish the fact that the foam has the same size as the black hole.

\section{CONCLUSIONS AND FUTURE DIRECTIONS}

We have explored the charges and angular momenta of a large family of smooth three-charge BPS geometries. We have found that, in the limit when the number of twocycles is large, a large subclass of these geometries have the correct charges, angular momenta, and size to be microstates of the three-charge maximally spinning BPS black hole in five dimensions.

A remarkable result of this analysis is that the upper bounds on the BPS black hole angular momenta, $J_{1}=$ $J_{2}=\sqrt{Q_{1} Q_{2} Q_{3}}$, which are classically found by requiring the absence of closed timelike curves on the black hole horizon, also emerge from the study of smooth horizonless solutions that represent black hole microstates. This is also not a special feature of $\mathrm{M}$ theory on $T^{6}$; it is also true in any $U(1)^{k}$ supergravity.

In fact, using a GH base with a large number of alternating plus and minus centers localized in a single "blob,"

\footnotetext{
${ }^{5}$ Nevertheless, it is quite possible that there might exist a "small black hole" whose entropy matches (5.3) or (5.5).
}

and with a well-behaved distribution of flux parameters, it is hard to obtain anything but maximally spinning BPS black hole microstates. It would be interesting to try to see if one can obtain microstate geometries with $J_{1}=J_{2}<$ $\sqrt{Q_{1} Q_{2} Q_{3}}$ using a GH base. It is possible that such solutions might only be obtained using a less-constrained (i.e. not Gibbons-Hawking) four-dimensional hyper-Kähler manifold as a base.

All the solutions obtained here can be easily dualized to a frame where they have D1, D5, and P charges, and can be made asymptotic to $\mathrm{AdS}^{3} \times S^{3} \times T^{4}$ [23]. Hence, they are dual to states of the D1-D5 CFT. It is very important to try to understand what these CFT states are; this might allow us to find whether the typical black hole CFT microstates are dual to bubbling solutions.

Another interesting result of our investigation is that the microstates of a zero-entropy black ring and the microstates of a zero-entropy black hole are all part of one and the same family: One can simply interpolate between them by moving a $\mathrm{GH}$ center with charge +1 away from the other GH centers, and there is no clear boundary between them. The fact that black ring and black hole microstates belong to the same family of solutions is very satisfying from the perspective of the microphysics of black objects. It also indicates that the dual states in the D1-D5 CFT are very similar, and this might represent an important step towards finding what these states are.

A third result of our investigation is that there are two significant contributions to the entropy of solutions: The topological entropy and the entropy from the moduli space. Our rough estimates suggest that the latter will dominate and that a typical geometry will have the size of the black hole, but will contain Planck-size bubbles. On the other hand, it is possible that our estimates were rather too simplistic. If the topological entropy were dominant, then the typical geometry would have bubbles and curvatures parametrically lower than the Planck scale. It is therefore very important to understand how to address the quantization of this moduli space.

It should also be remembered that our analysis here is based upon geometries with a Gibbons-Hawking base metric, which are a very special class of the allowed hyper-Kähler base metrics. This was done primarily because they enable us to perform explicit computations. From the physics of supertubes $[7,11,24]$ we expect more general hyper-Kähler metrics to involve a large number of arbitrary functions [1], which should give a contribution to the entropy much larger than the topological and the moduli space entropies. It is very important to build and understand these metrics, and to find whether this hyperKähler entropy favors large or small bubbles.

Finally, this work provides a starting point from which one can perform an entropy calculation of the black hole microstates in the spirit of $[25,26]$, using perhaps the techniques of $[27,28]$ which could prove or disprove 
Mathur's conjecture. Such calculations are usually done by perturbing around classical zero-entropy configuration and, prior to the our bubbled solutions, no such black hole microstates were known. This obstacle to progress has now been eliminated.

\section{ACKNOWLEDGMENTS}

We would like to thank Juan Maldacena, Don Marolf, Per Kraus, Slava Rychkov, and Davide Gaiotto for inter- esting discussions. I. B. would like to thank the Kavli Institute for Theoretical Physics for hospitality during part of this work. The work of N.W. and C.-W.W. is supported in part by the DOE grant No. DE-FG03-84ER40168. The work of I. B. is supported in part by the NSF grants No. PHY-0503584 and No. PHY-990794.
[1] I. Bena and N. P. Warner, Phys. Rev. D 74, 066001 (2006).

[2] P. Berglund, E. G. Gimon, and T. S. Levi, J. High Energy Phys. 06 (2006) 007.

[3] S. D. Mathur, Fortschr. Phys. 53, 793 (2005).

[4] I. Bena and N.P. Warner, in Proceedings of the Winter School on the Attractor Mechanism, Frascati, Italy, 2006 (Springer, New York, to be published).

[5] J.P. Gauntlett, J. B. Gutowski, C. M. Hull, S. Pakis, and H. S. Reall, Classical Quantum Gravity 20, 4587 (2003).

[6] J. B. Gutowski and H. S. Reall, J. High Energy Phys. 04 (2004) 048.

[7] I. Bena and N.P. Warner, Adv. Theor. Math. Phys. 9, 1 (2006).

[8] J.P. Gauntlett and J.B. Gutowski, Phys. Rev. D 71, 045002 (2005).

[9] S. Giusto, S. D. Mathur, and A. Saxena, Nucl. Phys. B710, 425 (2005); S. Giusto and S. D. Mathur, Nucl. Phys. B729, 203 (2005).

[10] J. C. Breckenridge, R. C. Myers, A. W. Peet, and C. Vafa, Phys. Lett. B 391, 93 (1997)

[11] I. Bena and P. Kraus, Phys. Rev. D 70, 046003 (2004).

[12] H. Elvang, R. Emparan, D. Mateos, and H. S. Reall, Phys. Rev. Lett. 93, 211302 (2004).

[13] H. Elvang, R. Emparan, D. Mateos, and H. S. Reall, Phys. Rev. D 71, 024033 (2005).
[14] S. Giusto, S. D. Mathur, and Y. K. Srivastava, Nucl. Phys. B763, 60 (2007).

[15] F. Denef, J. High Energy Phys. 08 (2000) 050.

[16] B. Bates and F. Denef, hep-th/0304094.

[17] I. Bena and P. Kraus, Phys. Rev. D 72, 025007 (2005).

[18] A. Saxena, G. Potvin, S. Giusto, and A. W. Peet, J. High Energy Phys. 04 (2006) 010.

[19] I. Bena, C. W. Wang, and N. P. Warner, J. High Energy Phys. 11 (2006) 042.

[20] R. Kallosh, A. Rajaraman, and W. K. Wong, Phys. Rev. D 55, R3246 (1997).

[21] F. Denef, J. High Energy Phys. 10 (2002) 023.

[22] I. Bena, C. W. Wang, and N. P. Warner, J. High Energy Phys. 05 (2006) 075.

[23] I. Bena and P. Kraus, J. High Energy Phys. 12 (2004) 070.

[24] I. Bena, C. W. Wang, and N. P. Warner, J. High Energy Phys. 03 (2006) 015.

[25] B. C. Palmer and D. Marolf, J. High Energy Phys. 06 (2004) 028.

[26] D. Bak, Y. Hyakutake, and N. Ohta, Nucl. Phys. B696, 251 (2004); D. Bak, Y. Hyakutake, S. Kim, and N. Ohta, Nucl. Phys. B712, 115 (2005).

[27] L. Grant, L. Maoz, J. Marsano, K. Papadodimas, and V. S. Rychkov, J. High Energy Phys. 08 (2005) 025.

[28] V.S. Rychkov, J. High Energy Phys. 01 (2006) 063. 